\title{
De la maison à la métalepse daiglienne
}

Benoit Doyon-Gosselin

Université de Moncton

Étais-je en train de me saboter moi-même?

Je ne voulais pas cela.

Pour sûr, p. 320.

Dans le corpus romanesque de la littérature acadienne, la question de la représentation spatiale de l'écrivain et de l'écriture se pose selon des modalités complexes dans l'œuvre d'une auteure en particulier : France Daigle. Déjà en 2004, à l'occasion de la parution d'un numéro de la revue Voix et Images (2004) consacré à la romancière, les chercheurs résumaient les quatre 
lignes de force d'une œuvre atypique. Le dossier se penchait sur le rapport à la langue (Boudreau), les structures formelles (Paré), l'autofiction et ses conséquences (Den Toonder) et la spatialité (Doyon-Gosselin et Morency). J'aimerais laisser de côté la question de la langue, qui a certes pris une place exponentielle de Pas pire (2002a [1998]) à Pour sûr (2011) et qui, par conséquent a été analysée par quelques jeunes chercheurs (RobidouxDaigneault et Thivierge) et par des chercheurs établis (Boudreau, 1999 et 2000; Leclerc, 2006 et 2009). Il me semble toutefois qu'une réflexion sur les liens entre la spatialité, les structures formelles et l'autorepré-sentation de l'écrivain mérite d'être approfondie, surtout à la lumière de la parution de Pour sûr. Mon hypothèse est que les différentes autoreprésentations de l'écrivain dans l'œuvre de France Daigle demeurent tributaires de l'organisation spatiale et formelle des romans.

Pour évaluer la justesse de cette hypothèse, il faut dans un premier temps montrer comment, dès la parution de ses premiers romans dans les années 1980, l'utilisation de la figure spatiale de la maison sert à réfléchir à l'œuvre littéraire en construction. De plus, la présence de personnages représentant des créateurs - l'architecte par exemple - témoigne d'une autoreprésentation décalée, d'une auctorialité dialectisée en figures métaphoriques. Pour y arriver, les romans Sans jamais parler du vent (2013 [1983]), Film d'amour et de dépendance (2013 [1984]) et La Beauté de l'affaire (2016 [1991]) seront analysés brièvement. Dans un deuxième temps, il faudra expliquer comment le choix de se libérer des figures spatiales analogiques - conséquence de la victoire sur l'espace de la narratrice dans Pas pire -, associé à la complexification des contraintes formelles, permet au final une métalepse de l'auteur fort révélatrice dans Pour sûr. 


\section{Les lieux usés de la création et les avatars du créateur}

Dans son livre intitulé L'Espace et le sens. Germinal d'Émile Zola (1985), Denis Bertrand développe des thèses intéressantes et novatrices pour étudier les topoï. D'emblée, Bertrand propose que «la spatialité, aux divers niveaux où il est possible de la reconnaître et de la saisir, fonctionn[e] comme un principe organisateur supportant, de manière remarquablement systématique, le déploiement de plusieurs discours différents mais homologues » (p. 58). Pour ce faire, il s'applique à montrer que «les deux fonctions représentatives régies par les relations spatiales, fonction figurative et fonction abstraite, s'appuient l'une sur l'autre, se réfléchissent l'une l'autre, se fondent et se stipulent réciproquement» (p. 61). La fonction figurative se limite à traiter des topoï au premier degré. Un sujet, qu'il soit narrateur ou personnage, sélectionne ou met l'accent sur des objets spatiaux selon son point de vue. Chaque lieu décrit dans une œuvre, chaque topos permet de mieux saisir la relation entre le sujet et la construction spatiale d'une œuvre spécifique. En effet, pour Bertrand, il semble que

le parcours d'ensemble du sujet collectif s'éclaire de manière décisive dès lors qu'on le considère à travers les relations reconstructibles qui s'établissent et se transforment entre ce sujet et ses projections spatiales. En d'autres termes, son parcours spatial constitue le support signifiant de son parcours historique et social : le sujet "s'inscrit » et inscrit son devenir dans les figures des lieux. (p. 151)

La fonction abstraite des topoï suggère que ces derniers possèdent une signification au deuxième degré. Au-delà de leurs caractéristiques descriptives - de leur fonction figurative - les topoï sont porteurs de sens. À ce titre, Bertrand avance que 
par la place qu'occupent en son fonctionnement les catégories et les relations de l'espace, on peut affirmer que l'abstraction s'y structure comme figure, et que le discours abstrait, censé à la limite théoriser le sens, puise dans la spatialité certains des schèmes fondamentaux de sa construction. (p. 166)

Le discours abstrait repose alors sur les diverses relations spatiales dans une œuvre donnée. À partir d'un topos, de la place qu'il occupe par rapport aux autres topoï, le lecteur peut en arriver à comprendre un sens qui n'était pas néces-sairement apparent à première vue. Bref, de la fonction figurative dérive un sens qui trouve son accomplissement dans la fonction abstraite. De l'illusion du réel fourni par la fonction figurative, on passe à l'illusion de sens qui découle de la figure abstraite. La fonction figurative permet de décrire un élément spatial comme une maison ou une île, de cerner les différents traits de celui-ci. De son côté, la fonction abstraite permet d'intégrer l'objet spatial dans un système de signes plus vaste qui aide le lecteur à donner un sens à l'œuvre. C'est ainsi que fonctionnent les figures spatiales obsédantes dans les romans de France Daigle.

Dans Sans jamais parler du vent, premier roman de l'auteure, la figure de la maison n'est pas encore construite; il s'agit d'un projet: "La maison que l'on entend bâtir et qui sera un chefd'œuvre» (Daigle, 2013, p. 19). Elle constitue une figure hypothétique qui parcourt l'œuvre mais qui ne semble pas se concrétiser. Le narrateur souligne toutes les difficultés associées à ce projet de maison, et ce, à plusieurs reprises. Il devient difficile de vivre sans elle, de se situer dans l'espace : « La maison, la bâtir pour vivre» (Daigle, 2013, p. 125). En fait, la plus grande peur du narrateur se résume en peu de mots : « N'avoir plus de maison, plus lieu d'exister. » (Daigle, 2013, p. 132). La deuxième proposition de la phrase est révélatrice, car le lecteur s'attend plutôt à lire « plus de 
lieu pour exister ». De cette manière, le texte associe la maison à l'ontologie de l'être; sans maison, l'être ne pourrait exister.

Plusieurs critiques ont déjà souligné que «[1]e rapport métaphorique entre la maison à bâtir et le roman à faire [...] "contamine" de proche en proche d'autres motifs fictionnels et leur donne une portée métatextuelle » (Boudreau et Robichaud, 1988, p. 149-150). Cette " portée métatextuelle» permet de comprendre que toute l'œuvre de Daigle s'appuie sur la métaphore de la maison et de l'architecte. Un des problèmes inhérents à la construction de l'œuvre est qu'elle se construit elle-même, indépendamment de la volonté du narrateur : «La maison qui se construit, essayer parfois de l'habiter. Comme si ce n'était pas surtout elle qui nous habitait. Elle, parler encore d'elle. Comme si c'eût été possible qu'elle soit encore là » (Daigle, 2013, p. 31). À maintes reprises, le narrateur réitère son intuition qui lui fournit en fait une raison, ou plutôt une excuse pour poursuivre son projet littéraire. Ainsi, il est question «[d]es livres qui à bien y penser nous écrivent peut-être » (Daigle, 2013, p. 110) ou encore « [d] es premiers mots comme si le roman ne courait pas partout à notre rencontre de toute façon » (Daigle, 2013, p. 127). La construction de l'édifice romanesque devient alors un impératif hors du véritable contrôle du narrateur. Ainsi, il existe autant de possibilités de maisons que de romans, mais le narrateur parvient difficilement à mener à terme son projet, car il ne sait comment s'y prendre: "Ne plus savoir où aller pour écrire cette chose que tout continue pourtant à nous faire parvenir. » (Daigle, 2013, p. 36)

La métaphore filée de la maison pour l'œuvre est construite par analogies et répétitions. Chaque phrase qui traite de la maison évoque également, souvent dans la même proposition, le roman ou l'œuvre à venir. Le narrateur parle par exemple d'un long silence et 
d'un long voyage qui mène finalement à la maison : « Comme parfois au bout d'un très long silence comme au bout d'un très long voyage une maison, s'y arrêter » (Daigle, 2013, p. 85). La métaphore repose ici sur un extrait de la page suivante, qui reprend les mêmes mots, mais en précisant le lien entre la maison et le roman : «Le roman comme structure contre laquelle appuyer ses voyages, le cadre d'une porte. Les frontières alors, puis le devoir de rester en place. Les mots, les assaillir, les arrêter » (Daigle, 2013, p. 86). Le mot « roman », dans cet extrait, peut aussi bien être remplacé par le mot « maison » sans changer le sens de la phrase. Encore une fois, « le devoir de rester en place » et le fait d'utiliser des mots pour construire montrent bien l'urgence de dire, de nommer les choses. La maison devient alors un leitmotiv pour l'idée incessante du roman lui-même. Ainsi, il n'y a qu'un devoir essentiel pour le narrateur, obligation qui souligne encore toutes les connotations entre la maison et le roman: «Le roman, l'habiter absolument. Passer d'un lieu à un autre sans le temps qu'il faut normalement pour ces choses. » (Daigle, 2013, p. 82).

Dans le sillage de Sans jamais parler $d u$ vent, le deuxième roman de Daigle, Film d'amour et de dépendance, poursuit la quête de l'œuvre à construire en utilisant la figure de la maison et la présence de charpentiers, dans le but de réaliser un film (qui ne sera jamais achevé). La maison est au départ une simple image du film, mais son symbolisme déborde du cadre scénique. Ce n'est d'ailleurs pas sans raison que le narrateur affirme : «Les lieux de ce film n'étaient pas ceux que nous avions imaginés » (Daigle, 2013, p. 230). Cet énoncé indique clairement au lecteur qu'il ne faut pas comprendre la maison au premier degré, mais que ce topos signifie autre chose.

Le symbolisme de la maison témoigne de l'écriture autoréflexive de Daigle et manifeste surtout «que la seule construction dont il soit question dans ce livre est [...] celle du 
texte lui-même »(Boudreau, 1996, p. 77). Alors que le projet de départ semblait être un scénario de film, il devient peu à peu une réflexion sur le roman. Le narrateur doute et ne sait plus où il en est : « Ne plus savoir s'il s'agit de tourner un film ou de bâtir des maisons [...]. Des maisons un peu n'importe comment mais solides quand même, immuables » (Daigle, 2013, p. 202). Le parallèle entre la maison et l'œuvre semble évident. Film d'amour et de dépendance paraît bâti «un peu n'importe comment», mais fait partie, malgré tout, de ces jalons « immuables » de la littérature acadienne. De plus, la situation de l'écrivain évoluant en Acadie ressemble à celle des charpentiers dans le roman. Le narrateur propose en ce sens un rapprochement entre le peuple acadien et ses écrivains :

Ici les gens sont renfermés, repliés sur eux-mêmes. Ils vivent dans des sortes de terreaux, subissent la pensée comme un mal à endurer. [...] Les charpentiers leur ressemblent mais ils ne sont pas tout à fait de la même famille. Ils leur bâtissent des abris convenables et puis s'en vont. (Daigle, 2013, p. 212)

L'analogie entre les charpentiers et les écrivains s'appuie sur la métaphore de la maison comme œuvre. Dans ce passage, bien que les charpentiers (ou les écrivains) fassent partie de la société, ils n'ont aucune appartenance familiale. Après le travail de construction (ou d'écriture) accompli, au meilleur de leurs capacités, ils partent pour reprendre leur place en marge de la société. L'émergence de la figure de l'écrivain, sous les traits des charpentiers, permet au lecteur de mieux saisir la portée de leurs actions. Par exemple, le narrateur spécifie « que les charpentiers eux fouillent dans les entassements de bois livrés là pêle-mêle, retirent des morceaux qui font leur affaire » (Daigle, 2013, p. 188). Le charpentier, tout comme l'écrivain, « travaille avec un langage qui a déjà servi et qu'il a pour mission de renouveler, 
qu'il doit se réapproprier » (Boudreau et Robichaud, 1988, p. 150). Cette réappropriation langagière passe nécessairement par la réappropriation de l'espace. C'est d'ailleurs ce que personnages et narrateur tentent d'accomplir dans le roman $L a$ Beauté de l'affaire, qui clôt un premier cycle romanesque.

Ce court roman est coiffé d'un sous-titre révélateur: Fiction autobiographique à plusieurs voix sur son rapport tortueux au langage. Les deux premières propositions de ce sous-titre témoignent de l'autoreprésentation brouillée de France Daigle. Dans un premier temps, le caractère autobiographique de l'œuvre laisse entendre que la vie ou, à tout le moins, une fiction de la vie de France Daigle s'offrira au lecteur. Cette piste est pourtant fausse, car la romancière n'est pas un personnage du roman. La voix qui commente le texte, le pronom «elle», remplace le pronom personnel «je», auquel le lecteur est habitué dans une fiction autobiographique. Il s'agit du double fictionnel de l'auteure. Daigle s'inscrit donc dans son histoire en spécifiant qu'il s'agit d'une fiction autobiographique, mais s'en distancie simultanément en utilisant le pronom personnel « elle» au lieu du «je ». On comprend alors toute l'ambiguïté créée par le sous-titre.

Dans un deuxième temps, le sous-titre évoque «plusieurs voix » qui participeraient à l'élaboration de l'œuvre. Les voix en question se réfèrent aux trois trames narratives fragmentées qui s'ajoutent à la voix de la narratrice. La première trame narrative est celle d'un architecte qui prie, en compagnie de sa femme, à la messe de minuit. Quant à la deuxième trame narrative, elle met en scène un homme qui veut bâtir une clôture sur une île. Enfin, la troisième trame narrative est constituée d'un groupe d'artistes acadiens qui doit transformer un terrain vague en un « espace vert, agréable et 
séduisant » (Daigle, 2016, p. 89). Dans chaque trame narrative, les lieux diffèrent, mais il s'agit toujours d'investir un espace, d'y prendre place et de le rendre habitable. Les trois micro-récits mettent en scène la reprise analogique de la création littéraire. On peut également concevoir que l'architecte est un autre avatar du créateur et que France Daigle fait partie du groupe d'artistes fictifs qui travaille de façon «utile ». L'architecte, double de l'écrivain, tente de se projeter dans un espace structuré, cohérent, où les éléments disparates possèdent un sens : «Ainsi, où qu'il se trouve, l'architecte cherche des liens entre ce qui se présente à lui comme une foule d'éléments disparates. Il cherche une cohésion d'ensemble, et cette cohésion, quand il la trouve, il la projette dans l'espace» (Daigle, 2016, p. 119). En utilisant le personnage de l'architecte au lieu de l'écrivain, le narrateur peut ainsi réfléchir à l'espace en tant qu'analogie de l'écriture. En fait, il faut comprendre que tous les personnages sont des doubles de la voix narrative. Cette dernière possède des similitudes avec l'architecte, avec l'homme qui construit une clôture sur une île et également avec le groupe d'écrivains acadiens. Sur le plan symbolique, l'architecte représente le moment où l'écrivain cherche l'inspiration alors que l'homme dans son île représente le romancier seul devant son œuvre en construction tandis que le groupe d'écrivains acadiens suggère tous les rapports conflictuels qui surgissent dans le champ littéraire. La Beauté de l'affaire souligne de façon explicite l'importance du lien entre la création architecturale et la création littéraire dans un contexte universel: "Tous et toutes nous tripotons l'ordinateur tandis qu'ailleurs des gens sablent une planche, rabotent un poème, rassemblent des troncs, pour le papier. » (Daigle, 2016, p. 102)

Les différents topoï mis en scène par France Daigle dans la grande majorité de ses romans servent de support analogique à une 
réflexion sur la création littéraire. Ils accueillent les traits usés du créateur, sans pour autant mettre fin à la création. En utilisant plusieurs stratégies liées à la spatialité des œuvres, Daigle gomme sa présence sous les traits d'avatars. Cependant, l'intégration de personnages acadiens dans les romans subséquents, jumelée à la disparition progressive des topoï, analogon de la création, permettront à l'auteure de trouver sa place dans des structures formelles complexes.

\section{L'ultime transgression : la métalepse daiglienne}

En 2004, plus tardivement dans sa longue carrière de chercheur, Gérard Genette propose un petit ouvrage intitulé Métalepse. De la figure à la fiction. Genette y offre un historique de cette figure appartenant au domaine de la rhétorique pour ensuite se consacrer à une de ses acceptions plus modernes : la métalepse de l'auteur. Plus particulièrement, il présente maints exemples provenant du monde du cinéma en évoquant tout de même une des métalepses les plus célèbres de la littérature, celle de deux niveaux de narration incompatibles dans la nouvelle «Continuité des parcs» de Julio Cortázar. Pour Genette, la métalepse constitue une «manipulation - au moins figurale, mais parfois fictionnelle [...] - de cette relation causale particulière qui unit, dans un sens ou dans l'autre, l'auteur à son œuvre, ou plus largement le producteur d'une représentation à cette représentation elle-même » (p. 14). Évidemment, un auteur - par exemple Cortázar - peut créer une métalepse narrative, ce qui ne constitue pas en soi une métalepse de l'auteur comme celle qui m'intéresse. Cette métalepse particulière met en scène un «romancier entre deux romans, mais aussi entre son propre 
univers vécu, extradiégétique par définition, et celui, intradiégétique, de sa fiction » (Genette, p. 31).

Dans son œuvre, comme je l'ai montré plus haut, Daigle s'est servie de la figure de la maison pour parler de l'œuvre littéraire, de son œuvre en formation. Au fil des romans, des créateurs - architecte, charpentiers, artistes anonymes, peintre, etc. - qui, tous à leur manière, représentent l'écrivaine en devenir ont laissé leur place à un bébé, né en novembre 1953 à Moncton, même mois, même année et même lieu de naissance que France Daigle. Dans son roman 1953. Chronique d'une naissance annoncée (2014 [1995]), Daigle met en scène $a$ postériori comment un bébé a pu être influencé dans son développement par les événements locaux et mondiaux de son année de naissance. Elle s'approprie entre autres « le monde médical à des fins littéraires [afin de] naître à la littérature » (Daigle, 2014, p. 28). Cependant, on peut difficilement parler de métalepse dans ce cas. Même si le personnage du bébé deviendra plus tard écrivaine, son nom est tout simplement Bébé M. Comme le signale François Paré dans la préface de la réédition du roman, «Bébé M., c'est France Daigle elle-même, ou plutôt la part première qui échappe toujours à la romancière à l'affût de son histoire personnelle. » (Paré, 2014, p. 9) Il s'agit encore d'une projection de l'auteure.

Le roman suivant, intitulé Pas pire, présente trois trames narratives qui se rapprochent sans toutefois véritablement s'entrecroiser. On trouve tout d'abord l'histoire d'une écrivaine, explicitement nommée France Daigle, qui évoque son enfance à Dieppe et son agoraphobie, peur qu'elle affrontera lorsqu'elle se rendra en France pour obtenir le prix France-Acadie et pour participer à l'émission Bouillon de culture. Il s'agit de la trame narrative principale du roman. Deuxièmement, l'œuvre met en scène Terry Thibodeau et Carmen Després, un couple de jeunes Acadiens 
qui habitent la région de Moncton-Dieppe. Ce récit se concentre sur les hauts et les bas de cette relation amoureuse qui aboutit à la naissance prochaine d'un enfant. Enfin, une dernière trame narrative, de moindre importance, réunit Élisabeth, médecin habitant à Moncton, rencontrée pour la première fois dans La Vraie Vie (2016 [1993]), et Hans, qui avait aperçu Élisabeth en Grèce à la dernière page de 1953. Dans Pas pire, un narrateur omniscient relate les suites de cette union passagère et ses conséquences pour les deux personnages. Plus que tous les romans précédents de Daigle, le projet qui sous-tend Pas pire est lié à la spatialité problématique de l'auteure et de ses personnages : « Le projet consistait donc à écrire un livre portant très largement et très librement sur le thème de l'espace: espace physique, espace mental, et les façons que nous avons de nous y mouvoir » (Daigle, 2002a [1998], p. 54). À ces trois trames narratives, il faut ajouter tous les discours scientifiques (sur les deltas, sur la mythologie, les phobies, etc.) ou pseudo-scientifiques (sur l'astrologie, sur les rêves) intégrés à la diégèse.

Évidemment, la présence d'une fictionnalisation de Daigle dans le roman se rapproche d'une véritable métalepse de l'auteur. Surtout que, lorsqu'elle se rend en France pour rencontrer Bernard Pivot, l'écrivaine commente des passages du roman que le lecteur est en train de lire. En ce sens, le roman est méta- et autoréflexif. Pourtant, la narration prend bien soin de ne pas brouiller les trames narratives. France Daigle ne rencontre jamais ses personnages. Ils vivent chacun et chacune leur vie en parallèle. Ainsi, à l'aéroport, Daigle entrevoit Élizabeth, mais jamais ils n'auront de contacts autres que visuels :

Nous avancions dans un couloir tout en verre quand je [Daigle] vis venir, dans le sens contraire, une femme que je reconnaissais. $[\ldots]$ 
Juste comme nous la croisions, Élizabeth tourna la tête dans notre direction et nous aperçut la regardant. Elle sourit, quoiqu'un peu timidement. Quelques pas plus loin, hors de la portée de son regard, Camil ajouta :

-A doit pas savoir d'où c'est qu'on vient.

- Tant qu'à ça, je me le demande souvent moi-même. (Daigle, 2002a [1998], p. 172-173)

Ici, les trames se frôlent, mais ne se croisent pas. La rupture métaleptique est remise à plus tard.

En suivant Genette, on pourrait toutefois considérer que le roman propose une antimétalepse. En effet, si la métalepse est presque toujours conçue comme une «transgression ascendante, de l'auteur s'ingérant dans sa fiction (comme figure de sa capacité créatrice) » (Genette, p. 27), l'antimétalepse suggère que « sa fiction s'immisc[e] dans sa vie réelle» (p. 27). Dans Pas pire, Daigle met en scène une fictionnalisation d'elle-même qui se rend à Paris pour cueillir le prix France-Acadie et, au passage, participer à Bouillon de culture. Dans la vie réelle, l'écrivaine France Daigle n'avait jamais gagné le prix France-Acadie jusqu'à la parution de Pas pire, avec lequel elle gagne le prix ! Elle n'a cependant jamais participé à la populaire émission littéraire. On peut toutefois se demander pourquoi Daigle, dans une œuvre faisant la belle part à l'autofiction, se retient d'entremêler les fils narratifs. Une des raisons principales relève selon moi de la structure qui sous-tend l'œuvre. La presque totalité des romans de Daigle repose sur la présence d'une maison, itération spatiale par excellence pour aborder métaphoriquement la création littéraire. Cela étant dit, Pas pire est construit à partir d'une réflexion et des multiples utilisations du chiffre 12 :

le chiffre douze symbolise la complexité interne du monde [...] dynamique, représentant le perpétuel devenir de l'être et de l'univers. Nombre d'action, le chiffre douze représente aussi l'accomplissement et le cycle achevé. [...] Multiplié par lui-même, 
il mènerait à la plénitude et au paradis, rien de moins. (Daigle, 2002a, p. 99)

Le chiffre est omniprésent dans le roman (douze maisons astrologiques, douze diamants, douze travaux d'Hercule, etc.) et, malgré toute l'ironie de la dernière phrase du passage cité, il faut considérer que le roman repose sur la simple équation 12 fois 12 . Pas pire est en fait un roman en deux dimensions, un carré parfait dont l'aire serait 144. L'idée d'un roman en deux dimensions est renforcée par la présence d'une toile, Dénombrement de Bethléem de Bruegel l'Ancien, qui représente métaphoriquement le roman et les personnages qui vaquent à leurs occupations. En fait, l'impossibilité d'une métalepse de l'auteur dans Pas pire tient justement dans cette structure en deux dimensions. Dans le schéma suivant, la longueur du carré (l'horizontale) correspond à tous les discours scientifiques et pseudo-scientifiques alors que la largeur (la verticale) fait référence à l'histoire des personnages, à chaque trame narrative.

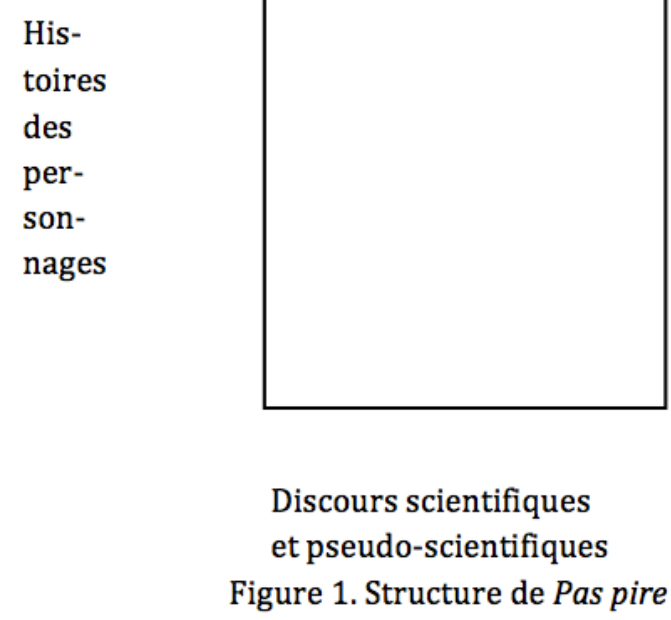


Bref, malgré toute la complexité du roman, Pas pire reste construit comme une maison dessinée par un enfant, en deux dimensions. Toutes ces considérations sont essentielles pour comprendre ce qui se passe entre la parution de Pas pire en 1998 et la parution de Pour sûr en 2011.

Dans ma monographie sur J.R. Léveillé et France Daigle, j'avais avancé (à tort, comme le montre Pour sûr) que « [d]ans Pas pire, la romancière France Daigle est elle-même architecte de son œuvre, mais en raison de sa victoire sur l'espace, elle élimine l'élément autoréflexif des romans subséquents » (DoyonGosselin, 2012, p. 352). Il est vrai que, dans Un fin passage (2001) et dans Petites difficultés d'existence (2002b), l'écrivaine ne met pas en scène une fictionnalisation d'elle-même. Certes, on pourrait dire que Zed, le jeune entrepreneur acadien qui engage un architecte et des ouvriers pour rénover les lofts, constitue un autre avatar de France Daigle, étant le créateur attitré du roman. L'ancienne usine abandonnée qui sera reconfigurée par Zed dans Petites Difficultés d'existence marque toutefois la dernière présence d'une figure spatiale servant de support à la réflexion de la littérature, réflexion tant créatrice que critique de l'autoreprésentation. Après ce roman, Daigle met de côté la figure spatiale analogique, le topos de son écriture, pour ne conserver qu'une structure numérale en prenant bien soin de l'exposer sans ambages. Cette mise au rancart des lieux usés de son œuvre laisse alors toute la place à l'écrivaine réelle (et non à sa fictionnalisation) sous la forme d'une métalepse ironique aux ramifications importantes.

Le roman Pour sûr, magnum opus de près de mille pages, comprend 144 trames narratives composées chacune de 12 fragments, ce qui donne au total 1728 fragments. Outre la mise en 
page du roman mettant l'accent sur cette structure, la présence d'un index, séparé de la table des matières, aide à remettre dans l'ordre numérique les différentes trames narratives. Une de ces trames, nommée à juste titre "Structure », rappelle dans son premier fragment que l'importance du chiffre douze débute dans Pas pire (Daigle, 2011, p. 55). Cependant, le deuxième fragment suggère que " [l]e 12 à la troisième puissance [...] paraît correspondre davantage aux exigences d'une plénitude ample et durable » (Daigle, 2011, p. 59). Ainsi, ces commentaires autoréflexifs sur la structure de l'œuvre confirment que, contrairement à Pas pire, qui était construit en deux dimensions (un carré), Pour sûr repose sur une construction en trois dimensions (un cube). Le simple fait que les fragments de chaque trame ne suivent pas, dans le livre, l'ordre prescrit par les numéros souligne, en dépit de sa forme rigide, le caractère désordonné du cube. Ainsi, il faut reprendre la figure proposée plus haut en deux dimensions et lui ajouter une troisième dimension. La longueur, à l'horizontale, forme la ligne temporelle de l'histoire collective de l'Acadie, plus précisément celle de Moncton et sa langue. La largeur, à la verticale, représente encore les histoires des différents person-nages qui s'ancrent dans le présent monctonien. Enfin, la profondeur est constituée de France Daigle, l'écrivaine acadienne qui ne se cache plus sous un avatar ou dans un lieu métaphorique de la création. Elle assume qu'elle a écrit d'autres romans auparavant et qu'elle est en train de bâtir ce roman. Comme elle fait partie de la troisième dimension du cube, elle peut traverser à son gré l'histoire collective acadienne et l'histoire de ses personnages sans complexe. Elle peut enfin procéder à une métalepse. 


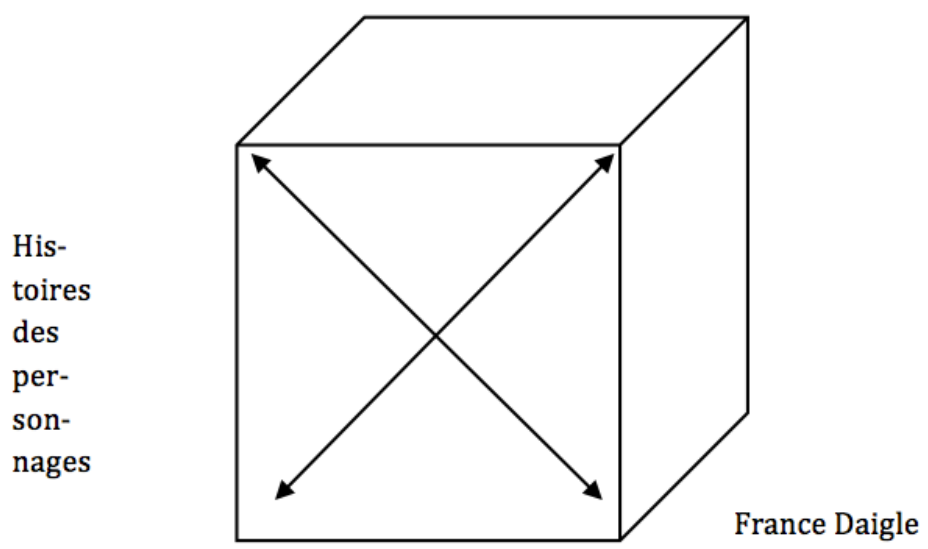

Histoire collective de l'Acadie

Figure 2. Structure de Pour sûr

Ce déplacement conscient entre les histoires des personnages, l'histoire de l'Acadie et la métaréflexion daiglienne sur son œuvre permet par exemple au roman de mettre en lumière les différentes stratégies utilisées par Daigle dans le passé. Dans la trame « Avatars », un narrateur (supposément) anonyme affirme : « Il ne serait pas inexact de dire que les personnages d'une fiction littéraire sont les avatars de son auteur, c'est-à-dire à la fois sa représentation, sa métamorphose et son malheur. » (Daigle, 2011, p.173) Éclairant ainsi son œuvre entière, Daigle offre une perspective sur la genèse du roman: «Premier titre envisagé : Jusqu'à la fin. Dans le contexte d'une description de projet pour une demande de bourse d'écriture au Conseil des Arts du NouveauBrunswick. Bourse obtenue, merci beaucoup.» (Daigle, 2011, p. 300). En privilégiant la structure du cube sans la lier à un topos analogique, Daigle ne voit plus la nécessité de se cacher derrière un avatar. Elle s'offre finalement toutes les possibilités narratives, dont celle de discuter avec ses personnages. 
La métalepse de l'auteur apparaît dans la trame narrative intitulée " Duos ». Il s'agit à chaque fois d'une rencontre et d'une discussion entre France Daigle et l'un de ses personnages. Le tableau suivant permet de schématiser ces rencontres :

\begin{tabular}{|c|c|c|}
\hline Fragment & $\begin{array}{c}\text { Personnage } \\
\text { rencontré }\end{array}$ & $\begin{array}{c}\text { Première apparition du } \\
\text { personnage dans les romans }\end{array}$ \\
\hline 538.101 .1 & Pomme & Petites Difficultés d'existence \\
\hline 650.101 .8 & Hans & $\begin{array}{c}\text { 1953. Chronique d'une } \\
\text { naissance annoncée }\end{array}$ \\
\hline 726.101 .2 & Ludmilla & Un fin passage \\
\hline 730.101 .3 & Zablonski & Un fin passage \\
\hline 972.101 .6 & Claudia & Un fin passage \\
\hline 1004.101 .7 & Carmen & Pas pire \\
\hline 1112.101 .12 & Shawn & Pour sûr \\
\hline 1150.101 .5 & Zed & Petites Difficultés d'existence \\
\hline 1180.101 .9 & Anonyme \# 2 & Pour sûr \\
\hline 1264.101 .11 & Sylvia & Petites Difficultés d'existence \\
\hline 1530.101 .10 & Josse & Petites Difficultés d'existence \\
\hline 1586.101 .4 & Terry & Pas pire \\
\hline
\end{tabular}

Sans analyser chaque rencontre, il faut tout de même prendre le temps de comprendre à quoi sert cette transgression ultime qui va au-delà du simple clin d'œil ironique. Pendant sa rencontre avec Hans, Daigle s'excuse et se justifie de l'avoir tenu à l'écart dans ses deux derniers romans incluant Pour sûr. Même si Daigle souhaite "unifier un peu [s]on univers", Hans comprend son rôle: "- Je ne suis pas comme tes autres personnages, n'est-ce pas ? Tu m'as créé pour te défaire de moi plus confortablement, c'est ça ? Ne réponds pas, les réponses sont toujours mauvaises le vendredi. » (Daigle, 2011, p 286) Le lecteur attentif remarquera le clin d'œil à l'obsession des jours qui habite Hans dans Un fin passage, mais surtout, le dialogue confirme que Daigle peut lire dans les pensées de ses personnages (normal : elle les a créés) et, réciproquement, les personnages 
peuvent lire dans les pensées de Daigle (anormal : ils sont des êtres de papier). D'ailleurs, lors de sa rencontre avec Zablonski, Daigle réitère l'idée que personnages et auteurs sont des vases communicants. Zablonski comprend que, lorsqu'il pense à un personnage rencontré dans un autre roman, Daigle y pense aussi : «Il avait raison, bien sûr, mais je ne voulais pas qu'il pense que tout ce qui lui arrivait m'arrivait aussi. » (Daigle, 2011, p. 320)

La métalepse de l'auteur se complexifie passablement dans les «duos» suivants. Dans une sorte de dédoublement de personnages, Daigle et Carmen vont manger dans un restaurant de Dieppe. La jeune femme dit à l'auteure : «-T'as vraiment eu une bonne idée de nous donner chacun une journée de brẽak de même. Ça nous fait vraiment du bien. » (Daigle, 2011, p. 432) La pause commune fait sourire ici. Daigle prend une pause de quoi ? De l'écriture? Est-ce une pause au sens genettien? Pause narrative, pause d'intrigue, on passe de l'action au métacommentaire mis en scène par l'apparition de la romancière. Le «nous » de l'extrait est véritablement un «je». D'ailleurs, Carmen laisse entendre qu'elle existe à cause de Daigle. Après que Carmen ait utilisé le mot «trébucher », Daigle dit : « - Trébucher. J'aime ce mot-là. » Ce à quoi Carmen répond : «C'est toi qui me l'as appris » (Daigle, 2011, p 433). Effectivement, dans Petites Difficultés d'existence, Carmen avait trouvé la façon standard de dire plusieurs mots qu'elle ne connaissait qu'en anglais à l'aide de dictionnaires visuels. Pourtant, Daigle n'est pas un personnage de ce roman et c'est dans la fiction uniquement que Carmen apprend de nouveaux mots. Ailleurs, quand Daigle explique à Zed qu'elle croyait qu'il se suiciderait après la fin du projet des lofts, ce dernier s'indigne. Il ne peut pas croire que l'auteure en avait décidé ainsi. Daigle avoue alors : « Je n’avais jamais pensé que mes personnages étaient conscients des attentes que j'avais à leur 
égard. En avais-je réellement ? » (Daigle, 2011, p. 496) Toutes ces rencontres métaleptiques constituent des chassés-croisés pendant lesquels l'auteure ne rejoint pas totalement son personnage, comme si les multiples avatars de l'auteure lui glissaient parfois entre les doigts.

La rencontre avec Anonyme \# 2 confirme cependant la place de l'auteure dans sa fiction et le contrôle qu'elle exerce sur celle-ci. Anonyme \# 2 rappelle en fait les premiers personnages daigliens, qui restent indéterminés : «Il ou elle fit la moue.» (Daigle, 2011, p. 511) Il s'agit d'un personnage neutre (voir Lefrançois), sans nom, sans sexe, comme dans ceux et celles présents dans Sans jamais parler du vent. De façon ironique, ce personnage est offusqué de se retrouver dans le roman en cours. Au nom de tous les personnages de l'œuvre de Daigle, il souhaite qu'elle demande la permission. Sinon, il serait prêt à la poursuivre en justice. Même si l'auteure est exaspérée par ces élucubrations, elle doit régler le problème au nom... de la fiction : «Décidément, j'aurais préféré être ailleurs, mais en même temps, je voulais à tout prix faire avancer mon livre » (Daigle, 2011, p. 512). Les doléances des personnages font sourire car, de toute évidence, ceux-ci n'existent qu'à cause de Daigle. Même quand Josse se fâche du fait que l'auteure part de fausses rumeurs qui portent sur Terry et Carmen, France Daigle met les pendules à l'heure : « De toute évidence, Josse oubliait que c'était d'abord moi qui les faisais vivre, eux, et elle aussi tant qu'à y être. » (Daigle, 2011, p. 652) Plus que jamais auparavant dans ses romans, l'auteure assure son rôle sans passer par une figure spatiale représentant l'œuvre et un avatar de l'écrivain. Alors que les premiers romans étaient des « livres qui à bien y penser nous écrivent peut-être » (Daigle, 2013, p. 110), où l'auteure s'avère plus passive qu'active, simple catalyseur de la fiction, 
Pour sûr est le roman assumé par Daigle. Ce constat est indéniable dans la dernière métalepse, lorsque Terry rencontre enfin Daigle.

Terry comprend peut-être pour la première fois qu'il n'est qu'un avatar de Daigle. Un peu surpris par cet état de fait, il s'exclame : "J'ai déjà entendu ça des écrivains dire que c'est les personnages qui finissont par prendre õver l'histoire, bưt c'est dur à crouère. Je veux dire, l'histoire tombe pas du ciel, faut que quelqu'un y pense » (Daigle, 2011, p. 679). Daigle avoue alors qu'elle n'est qu'un « filtre ». Filtre de quoi ? Elle ne le précise pas. On peut toutefois imaginer qu'elle agit comme un filtre entre la fiction et la réalité, qu'elle peut justement naviguer à son gré entre le Moncton de son époque et des personnages qui pourraient y habiter et qui, dans leur esprit, y habitent vraiment. La dernière remarque de Daigle s'avère essentielle pour comprendre le rôle de l'écrivain dans la fiction et dans la société : « En m'en allant, je me demandai s'il était normal de préférer des personnages aux vraies personnes. » (Daigle, 2011, p. 680). Dans un roman qui brouille les frontières entre personnages et vraies personnes, Daigle a choisi son camp. Ses personnages sont plus intéressants que la réalité et finissent par faire partie de sa réalité. Le filtre s'amincit et les avatars deviennent bien plus que la représentation, la transformation et le malheur de l'écrivaine. Ils sont La Vraie Vie, la seule qui importe vraiment. Si un jour, le narrateur daiglien avait affirmé que « [l]a conscience, celle d'un romancier ou d'une romancière s'interrogeant sur son art, par exemple, pourrait être considérée comme une anomalie » (Daigle, 2014, p.38), il est clair que cette anomalie est dorénavant assumée dans Pour sûr. 


\section{Conclusion}

Ce tour d'horizon de l'œuvre d'une romancière acadienne de première importance offre un exemple singulier des liens entre espace et topoï dans les romans de l'écrivain. Rappelons-le d'emblée : la figure spatiale de la maison est omniprésente dans les romans de France Daigle. Ce topos obsessif, plus qu'une métaphore filée, pourrait être considéré comme une métaphore filante qui traverse de part en part les contraintes formelles imposées par Daigle. La maison comme métaphore de l'œuvre littéraire est parfois remplacée par d'autres figures spatiales, comme un terrain vague ou une île, mais à chaque fois, il s'agit de réfléchir à la création littéraire. Qu'importe alors la fonction figurative des topoï quand, au final, c'est la fonction abstraite qui prend toute la place. Associés à cette spatialité créatrice, des avatars de l'écrivain peuplent les fictions de Daigle. Domestiques, charpentiers, architectes, ces personnages, anonymes au début, prennent une place de plus en plus importante pour penser la création. Ils agissent comme la conscience fictionnelle de l'auteure.

La complexification des structures formelles des romans daigliens à partir de Pas pire jusqu'à Pour sûr entraîne comme conséquence une lente disparition des topoï de la création. Alors que les avatars de l'écrivaine servaient de filtre entre les figures spatiales et la romancière, le dernier roman élimine la spatialité métaphorique pour laisser toute la place à une France Daigle qui construit le sens de l'œuvre en négociant avec ses personnages. Il n'existe plus de doutes sur la voix narrative et sur l'organisation du récit: Daigle gère avec assurance sa fiction. Dans l'absolu, elle n'offre peut-être pas un point de vue nouveau sur la création romanesque, mais à l'intérieur de son œuvre, la 
métalepse de l'auteure constitue une effraction majeure et un aboutissement logique. Ce contrôle narratif dans le cube formel permet toutes les libertés, comme une écriture du chiac inventé. Il permet enfin à France Daigle de créer son propre « Fictionnaire» (trame 120) où les mots remettent en cause la plénitude du $12^{3}$. Se jouant autant du lecteur et de sa fiction, Pour sûr est un exemple probant de

perfiction : n.f. - v. 2005 ; illusion de perfection. «Je suis désolée d'avoir à vous l'annoncer, mais vous vous êtes laissés berner par une sublime perfiction. » (Daigle), (Daigle, 2011, p. 456)

\section{Bibliographie}

BERTRAnd, Denis. (1985), L'Espace et le sens. Germinal d'Émile Zola, Paris, Hadès, coll. " Actes sémiotiques ».

Boudreau, Raoul et Anne Marie Robichaud. (1988), «Symétries et réflexivité dans la trilogie de France Daigle », Dalhousie French Studies, no 15, automne-hiver, p. 143-153.

Boudreau, Raoul. (1996), «Le silence et la parole chez France Daigle », dans Raoul Boudreau, Anne Marie Robichaud, Zénon Chiasson et Pierre M. Gérin (dir.), Mélanges Marguerite Maillet: recueil de textes de création et d'articles sur la littérature, la langue et l'ethnologie acadiennes en hommage à Marguerite Maillet, Moncton, Éditions d'Acadie, p. 71-81.

—. (1999), «L'hyperbole, la litote, la folie : trois rapports à la langue dans le roman acadien », dans Lise Gauvin (dir.), Les Langues du roman: Du plurilinguisme comme stratégie textuelle, Montréal, Presses de l'Université de Montréal, coll. « Espace littéraire », p. 73-86. 
-. (2000), "Les français de Pas pire de France Daigle», dans Robert Viau (dir.), La Création littéraire dans le contexte de l'exiguïté, 9e colloque de l'APLAQA, Beauport (Québec), MNH, coll. «Écrits de la francité », p. 51-63.

- . (2004), « Le rapport à la langue dans les romans de France Daigle : du refoulement à l'ironie », Voix et Images, vol. XXIX, $\mathrm{n}^{\circ} 3$, printemps, p. 31-45.

DAIGLE, France. (2013 [1983, 1984, 1985]), Sans jamais parler du vent, Film d'amour et de dépendance, Histoire de la maison qui brûle, Sudbury, Prise de parole, coll. « BCF ».

—. (2016 [1985, 1991, 1993]), Variations en B \& K, La Beauté de l'affaire, La Vraie Vie, Sudbury, Prise de parole, coll. « BCF ».

-. (2014 [1995]), 1953. Chronique d'une naissance annoncée, Sudbury, Prise de parole, coll. « BCF ».

—. (2002a [1998]) Pas pire, Montréal, Boréal, coll. «Boréal compact ».

- (2001), Un fin passage, Montréal, Boréal.

—. (2002b), Petites Difficultés d'existence, Montréal, Boréal.

—. (2011), Pour sûr, Montréal, Boréal.

DEN TOONDER, Jeanette (2004), « Dépassement des frontières et ouverture dans Pas pire », Voix et Images, vol. XXIX, $\mathrm{n}^{\circ} 3$, printemps, p. 57-68.

Doyon-Gosselin, Benoit et Jean Morency. (2004), « Le monde de Moncton, Moncton ville du monde : l'inscription de la ville dans les romans récents de France Daigle », Voix et Images, vol. XXIX, $n^{\circ} 3$, printemps, p. 69-83.

Doyon-Gosselin, Benoit (2012), Pour une herméneutique de l'espace : l'ouvre romanesque de J.R. Léveillé et France Daigle, Québec, Nota bene. 
GENETTE, Gérard. (2004), Métalepse. De la figure à la fiction, Paris, Seuil, coll. « Poétique ».

LECLERC, Catherine. (2006) «Ville hybride ou ville divisée : à propos du chiac et d'une ambivalence productive", Francophonies d'Amérique, ${ }^{\circ} 22$, p. 153-165.

- (2009) «L'Acadie (s')éclate-t-elle à Moncton? Notes sur le chiac et La distance habitée », dans Lucie Hotte et Guy Poirier (dir.), Habiter la distance. Études en marge de La Distance habitée, Sudbury, Prise de parole, coll. « Angora », p. 15-36.

LEFRANÇOIS, Émilie. (2005), «Le désir d'“écrire au neutre”: lecture des manifestations du genre dans les deux trilogies de France Daigle », mémoire de maîtrise, UQAR, 150 f.

PARÉ, François. (2004), «France Daigle: intermittences du récit », Voix et Images, vol. XXIX, n 3 , printemps, p. 47-55.

—. (2014), «Préface. 1953, de France Daigle. Portrait de la romancière acadienne à sa naissance », dans France Daigle (2014 [1995]), 1953. Chronique d'une naissance annoncée, Sudbury, Prise de parole, coll. « BCF», p. 7-15.

Robidoux-Daigneault, Camille. (2015), « De Pas pire à Pour sûr. Fait et effets des langues chez France Daigle », mémoire de maîtrise, Université de Montréal, 87 f.

ThiviERGE, Jessica. (2016), « Espaces et langues (dé)cloisonnés dans les quatre derniers romans de France Daigle », mémoire de maîtrise, Université McGill, $115 \mathrm{f}$.

\section{Résumé}

Cet article traite de l'œuvre romanesque de l'écrivaine acadienne France Daigle à partir d'une réflexion sur les liens entre la spatialité, les structures formelles et l'autoreprésentation de l'écrivaine qui trouve son aboutis-sement avec la parution de 
Pour sûr (2011). Il s'agit de montrer comment, dès la parution de ses premiers romans dans les années 1980, l'utilisation de la figure spatiale de la maison sert à réfléchir à l'œuvre littéraire en construction. De plus, il faut insister sur la présence de personnages représentant des créateurs - l'architecte par exemple -, qui témoigne d'une autoreprésentation décalée, d'une auctorialité dialectisée en figures métaphoriques. Ensuite, l'article montre comment le choix de se libérer des figures spatiales analogiques, associé à la complexification des contraintes formelles, permet de proposer une métalepse de l'auteure fort révélatrice dans le dernier roman publié à ce jour.

\section{Abstract}

This article deals with the work of the Acadian writer France Daigle, based on the links between spatiality, formal structures and the self-representation of the writer, culminating in the publication of the novel Pour sûr (2011). In the first part, the objective is to show that, when Daigle's first novels were published in the 1980s, the use of the spatial figure of the house serves to reflect on the literary work under construction. In addition, we must insist on the presence of characters representing creators - the architect for example - who testifies to a shifted autorepresentation, an auctoriality dialectized into metaphorical figures. Then, in the second part, the article shows how the choice to free oneself from the analogical spatial figures, associated with the complexification of the formal constraints, makes it possible to propose a metalepsis of the author who shines a light on the process of creation in the last novel published to this day. 